\title{
A head-mounted display holder for eye movement recording
}

\author{
CALVIN F. NODINE and JAMES J. McGINNIS \\ Temple University School of Medicine, Philadelphia, Pennsylvania 19140
}

\begin{abstract}
A lightweight head-mounted display holder is described which eliminates the need to hold the head fixed during eye movement recording. A comparison of this device with a standard fixed-head condition indicates that performance is comparable under the two conditions but that subjects report less discomfort with the head-mounted display holder.
\end{abstract}

Most methods for recording eye movements require the head to be fixed. This is usually accomplished using a head holder or bite board. Even with such head-restricting devices, head movements occur and contribute to error in locating the locus of gaze. Children and some special subject populations such as psychiatric patients are especially prone to error. Even experienced subjects may have difficulty holding the head still during recording trials of more than $30 \mathrm{sec}$. These subjects often report that head-holding devices are uncomfortable.

With these points in mind, we undertook to develop a device for holding the display still rather than the head. The head-mounted display holder that we developed was fashioned from a physician's ENT headband, which normally has a mirror mounted on it. We removed the mirror and attached a lightweight superstructure for holding stimulus cards ranging from $7.6 \times 10.2 \mathrm{~cm}$ ( $3 \times 4$ in.) to $20.3 \times 25.5 \mathrm{~cm}(8 \times 10 \mathrm{in}$.).

\section{DESCRIPTION OF APPARATUS}

The head-mounted display holder consists of an aluminum superstructure for holding stimulus cards attached to a physician's standard white plastic headband (PYNCHON). The headband is adjustable and rigid enough to hold the lightweight display assembly. For additional support, nylon straps that form a "cap" over the top of the head are added once the display assembly is attached to the headband. Figure 1 shows the head-mounted display holder worn by a subject.

The display assembly is made from lightweight aluminum C-shaped channel rods (Reynolds No. 6012) purchased from a local hardware center. The channel rods are fashioned into a $v$ shape and bolted to a single center rod; at the end of the rod is glued a $4 \times 6$ in.

This rescarch was supported by DAAG Grant 29-76G0313. Address requests for reprints to the authors, Radiology Research Laboratory, Temple University School of Medicine. Philadelphia, Pennsylvania 19140. black foam board and paper clips to hold stimulus display cards. The display assembly is bolted to the headband at three points: the center and the left and right temples. For added stability, three additional channel rods are bolted to the headband and fastened together above the head to form a solid isolage triangle. A fourth channel rod is bolted to the center rod at the $\mathrm{V}$ on the display assembly. The pyramidal shape of the superstructure provides rigidity and allows the headband to be adjusted for different head sizes. The assembly is counterbalanced by a fifth channel rod attached by rubber bands so that it can be adjusted to compensate for the size of the stimulus card. An adjustable head "cap" consisting of four 1/2-in. nylon-web straps is sewn together and attached to the headband. Foam rubber padding is sewn to the headband at the center and temple junctions with the display assembly and at the rear junction. The entire headgear weighs only $380 \mathrm{~g}$.

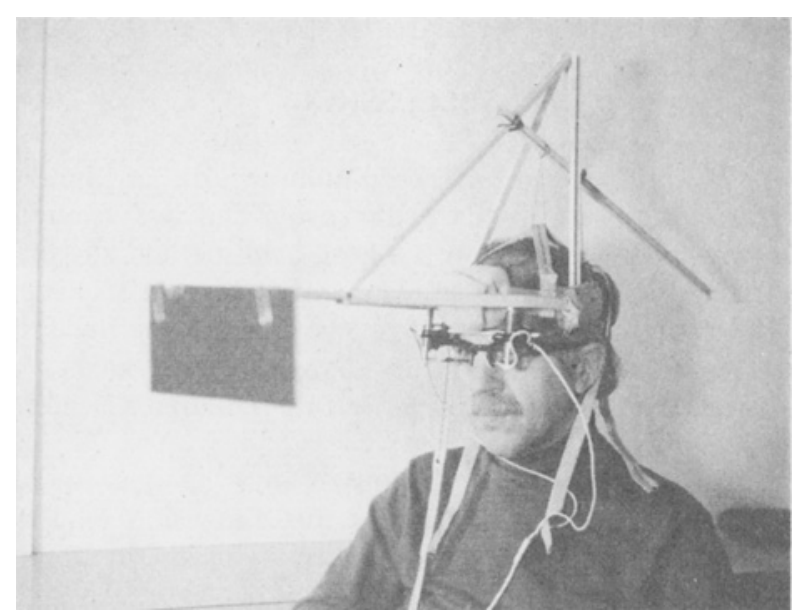

Figure 1. Subject with head-mounted display holder for cye movement recording. 
Table 1

Comparison of Accuracy (in Degrees) of Eye Movement Data Obtained Under Fixed-Head and Fixed-Display Conditions

\begin{tabular}{|c|c|c|c|c|c|c|}
\hline & \multicolumn{6}{|c|}{ Accuracy } \\
\hline & \multicolumn{3}{|c|}{$\mathrm{x}$ Axis } & \multicolumn{3}{|c|}{ y Axis } \\
\hline & Mean & SD & SE & Mean & SD & SE \\
\hline Fixed Head Using Head Holder & .38 & 1.05 & .10 & .40 & 1.15 & .12 \\
\hline Fixed Display Using Head-Mounted Display Holder & .13 & 1.27 & .13 & .67 & .88 & .09 \\
\hline
\end{tabular}

Note-Based on data from three subjects. Each subject looked at each of the five display points three times. The means are based on approximately 100 fixations. Under these viewing conditions, $1 \mathrm{deg}=.71 \mathrm{~cm}$.

When the headgear is worn, the display is held approximately $45 \mathrm{~cm}$ in front of the subject. At this distance, $1 \mathrm{~cm}$ equals $1.3 \mathrm{deg}$.

\section{PERFORMANCE OF THE APPARATUS}

The head-mounted display holder was tested by three experienced subjects who looked at the corners and center of a 5 by 5 dot matrix under two conditions: with the headgear and with the head restrained by a head holder. Eye movements were recorded with a limbus reflection system (Biometrics eye movement monitor) interfaced to a PDP $11 / 40$ computer.

Table 1 indicates accuracy in the fixed-display condition was somewhat better in $\mathrm{x}$ and poorer in $y$ than in the fixed-head condition. Accuracy is defined as the mean difference between the locus of gaze and the true position of the target dot in both $x$ and $y$ directions. The magnitude of these mean differences in terms of visual angle is within $2 / 3 \mathrm{deg}$. This means that the accuracy of the system under either condition was, at worst, $.84 \mathrm{deg}$ (mean difference of head-mounted y $\pm 2 \mathrm{SE}$ ), which, for a stimulus on a $20.3 \times 25.4 \mathrm{~cm}$ $(8 \times 10$ in.) display, translates to about $3 \%$ error.

\section{DISCUSSION}

The finding that the head-mounted display holder is more accurate in the $\mathrm{x}$ dimension and less accurate in the $y$ dimension than the head-held method suggests that the present device may be useful for tasks requiring close monitoring of the $\mathrm{x}$ axis. The comparative freedom of head movement afforded by this device makes it particularly useful for monitoring children's reading behavior.

Accuracy in the $x$ dimension may be due to the fact that turning the device in this dimension requires rotation of it about the head. Since the plastic headband fits snugly against the head, the unit is difficult to turn along the $x$ axis. Contraction of the forehead muscles causes slippage of the headband on the $y$ axis.

Subjects report that the headgear is comfortable over prolonged periods of viewing (i.e., $1 / 2 h$ ). One reason is that the subject has the freedom to choose a comfortable position during eye movement runs. Since there is no bite board or chin rest, subjects are able to talk between runs. Further tests are necessary to determine the effects of talking during the eye movement runs.

In addition to allowing the subject to talk, the absence of a bite board and chin rest simplifies the set-up for the eye movement run. Initial placement of the headgear is not critical because the precalibration run is performed after the headgear is adjusted.

Alternate solutions to the head movement problem have been outlined by Young and Sheena (1975). One solution is to use a series of light-emitting diodes to drive detectors that in turn signal a computer. Another system uses multiple ultrasonic receivers mounted on a helmet to determine head movement by measuring sonic delay time between the receivers and a triangular array of transmitters mounted some distance in front of the helmet. While the accuracy of these devices is good $( \pm 1 \mathrm{deg})$, they are considerably more complex and costly than the present device, which costs less than $\$ 20$ in materials.

We believe that future designs might capitalize on aspects of both the present design and that of Johnston and Hutton (1979). If the rigidity and light weight afforded in the present design were combined with the inherent stability of a more rigid headgear (e.g., the welder's headgear of Johnston and Hutton), the resultant device might provide better accuracy in both the $\mathrm{x}$ and $\mathrm{y}$ dimensions than fixed-head devices.

\section{REFERENCES}

Johnston, C. W., \& Hutron, T. J. A head-mounted scene holder for eye movement research. Behavior Research Methods \& Instrumentation, 1979, 11, 422-426.

Young, L. R., \& Sheena, D. Survey of eye movement recording methods. Behavior Research Methods \& Instrumentation, 1975, 7,397-429.

(Received for publication May 2, 1979; revision accepted May 15, 1979.) 